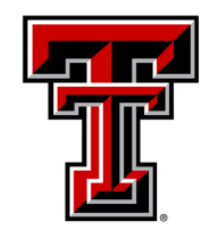

TEXAS TECH UNIVERSITY

Libraries"

\title{
LOCK-ON PHYSICS IN SEMI-INSULATING GAAS: COMBINATION OF TRAP-TO-BAND IMPACT IONIZATION, MOVING ELECTRIC FIELDS AND PHOTON RECYCLING
}

\section{The Texas Tech community has made this publication openly available. Please share how this access benefits you. Your story matters to us.}

\begin{tabular}{|l|l|}
\hline Citation & $\begin{array}{l}\text { A. R. Chowdhury, J. Dickens, A. Neuber, R. Ness, and R. P. Joshi, } \\
\text { "Lock-On Physics in Semi-Insulating GaAs: Combination of Trap-to- } \\
\text { Band Impact lonization, Moving Electric Fields and Photon } \\
\text { Recycling," Journ. Appl. Phys. 123, 085703 (2018). } \\
\text { https://doi.org/10.1063/1.5013248 }\end{array}$ \\
\hline Citable Link & $\underline{\text { https://hdl.handle.net/2346/86900 }}$ \\
\hline Terms of Use & $\begin{array}{l}\text { This article may be downloaded for personal use only. Any other } \\
\text { use requires prior permission of the author and AIP Publishing. }\end{array}$ \\
\hline
\end{tabular}




\section{Lock-on physics in semi-insulating GaAs: Combination of trap-to-band impact ionization, moving electric fields and photon recycling}

Cite as: J. Appl. Phys. 123, 085703 (2018); https://doi.org/10.1063/1.5013248

Submitted: 12 November 2017 . Accepted: 06 February 2018 . Published Online: 26 February 2018

A. R. Chowdhury, J. C. Dickens, (D) A. A. Neuber, R. Ness, and R. P. Joshi
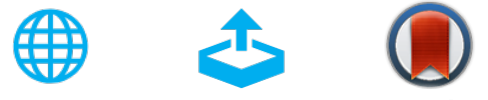

\section{ARTICLES YOU MAY BE INTERESTED IN}

Assessing the role of trap-to-band impact ionization and hole transport on the dark currents of $4 \mathrm{H}-\mathrm{SiC}$ photoconductive switches containing deep defects

Journal of Applied Physics 120, 245705 (2016); https://doi.org/10.1063/1.4972968

Investigation on properties of ultrafast switching in a bulk gallium arsenide avalanche semiconductor switch

Journal of Applied Physics 115, 094503 (2014); https://doi.org/10.1063/1.4866715

30kV and 3kA semi-insulating GaAs photoconductive semiconductor switch

Applied Physics Letters 92, 043511 (2008); https://doi.org/10.1063/1.2838743

Challenge us.

What are your needs for periodic signal detection?

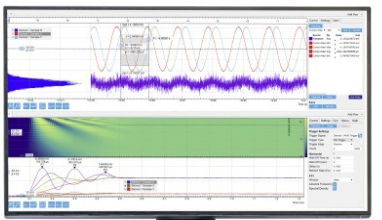

-
Zurich

- Instruments 


\title{
Lock-on physics in semi-insulating GaAs: Combination of trap-to-band impact ionization, moving electric fields and photon recycling
}

\author{
A. R. Chowdhury, ${ }^{1}$ J. C. Dickens, ${ }^{1}$ A. A. Neuber, ${ }^{1}$ R. Ness,${ }^{2}$ and R. P. Joshi ${ }^{1, a)}$ \\ ${ }^{1}$ Department of Electrical and Computer Engineering, Texas Tech University, Lubbock, Texas 79409, USA \\ ${ }^{2}$ Ness Engineering, Inc., P.O. Box 261501, San Diego, California 92196, USA
}

(Received 12 November 2017; accepted 6 February 2018; published online 26 February 2018)

\begin{abstract}
The time-dependent photoconductive current response of semi-insulating GaAs is probed based on one-dimensional simulations, with a focus on the lock-on phenomenon. Our results capture most of the experimental observations. It is shown that trap-to-band impact ionization fuels local field enhancements, and photon recycling also plays an important role in pushing the device towards lock-on above a $3.5 \mathrm{kV} / \mathrm{cm}$ threshold field. The results compare well with actual data in terms of the magnitudes, the rise times, and the oscillatory behavior seen at higher currents. Moving multiple domains are predicted, and the response shown depended on the location of the photoexcitation spot relative to the electrodes. Published by AIP Publishing. https://doi.org/10.1063/1.5013248
\end{abstract}

\section{INTRODUCTION}

Photoconductive switching is of interest for various electronic applications in the pulsed power arena, ${ }^{1-8}$ including microwave and millimeter wave generation, impulse and ultrawideband radar, particle accelerators, and directed energy systems. Advantages of optical switches include: (a) ultrafast response and turn-on times in the picosecond regime limited only by the characteristics of the optical trigger, (b) jitter-free response, (c) isolation between the electrical and optical systems, (d) superior repetitive rates, (e) higher-frequency response and controlled wave-shaping capability, (f) ability to scale to large voltages and currents in a single device without sacrificing speed, and $(\mathrm{g})$ potential selectivity between multiple optical trigger signals based on the wavelength-dependent response of photoconductive switches. For high power applications, semi-insulating GaAs (SI-GaAs) including $\mathrm{Cu}$ - or Fe-doped GaAs materials compensated with $\mathrm{Si}$-donors ${ }^{9}$ or low-temperature grown $\mathrm{GaAs}^{10}$ are often used as switch elements.

Experimental observations of GaAs photoconductive semiconductor switches (PCSS) have shown the presence of nonuniformities in the electric field, ${ }^{11}$ filamentary current flow when operated at fields above $\sim 10 \mathrm{kV} / \mathrm{cm}$ (Ref. 12) and a "Lock-On" effect. ${ }^{13}$ lock-on, which occurs when the switch is operated above a characteristic average electric field, results in a continuous flow of current even after the termination of the external optical pulse. This current endures over hundreds of nanoseconds. On the other hand, in the absence of lock-on, the GaAs photoconductive switch would typically be observed to open (i.e., cease to be in the conducting mode) very quickly, within the nanosecond timeframe. This feature seems to be universal in such semi-insulating direct bandgap photoconductive switches and has been reported in other materials such as InP, as well. ${ }^{1}$ In GaAs, electric fields roughly above $\sim 4 \mathrm{kV} / \mathrm{cm}$ were seen to be required for the onset of lock-on, while higher fields around $14 \mathrm{kV} / \mathrm{cm}$ were

\footnotetext{
a)Author to whom correspondence should be addressed: ravi.joshi@ttu.edu
}

reportedly needed for InP materials. ${ }^{14}$ Also, beyond the lock-on fields, the amount of light required to close the switch has been universally seen to reduce from the amount needed at low fields. Lock-on, therefore, has been associated with some internal gain mechanism.

Numerous theories have attempted to explain the lockon mechanism. Band-to-band impact ionization by electrons is one process that has been explored. ${ }^{15-17}$ Ionization rates for electron initiated band-to-band ionization in GaAs were fit to expressions stemming from the theories originally proposed by Crowell and $\mathrm{Sze}^{18}$ and Shockley. ${ }^{19}$ However, detailed calculations of band-to-band impact ionization on the basis of a realistic wavevector-dependent GaAs bandstructure $^{20,21}$ have shown that impact ionization at fields around $4 \mathrm{kV} / \mathrm{cm}$ is negligible. A simple curve fit of the fielddependent ionization coefficient $\alpha(F)$ from the band-to-band impact ionization calculations ${ }^{20}$ yields the following expression: $\alpha(F)=1.202 \times 10^{6} \exp \left(-1.5 \times 10^{6} / F\right)$, with $F$ denoting the electric field in $\mathrm{V} / \mathrm{cm}$. So, at electric field values around $10 \mathrm{kV} / \mathrm{cm}$ (taking a conservatively higher magnitude in comparison to the $4 \mathrm{kV} / \mathrm{cm}$ lock-on threshold), the curve-fit yields an ionization coefficient $\alpha(F)$ of almost zero. This low value indicates a negligible role for band-to-band ionization with regard to the lock-on phenomenon.

Another possible mechanism put forward to resolve the lock-on quandary was based on the negative differential mobility of electrons in $\mathrm{GaAs}^{22-24}$ which gives rise to Gunn domains associated with satellite-valley transfers. ${ }^{15,25-27}$ However, any launch of a Gunn domain requires fluctuations in either the electric field or carrier density near the cathode, which could be fashioned by doping non-uniformities that are either deliberate (for example, a "notch" structure) or through random imperfections. In semi-insulating materials, doping is typically avoided, and so the creation of such domains would not seem easy. Furthermore, for stable domain formation, the transit time needs to be greater than the dielectric relaxation time, leading to the Kroemer condition, ${ }^{28}$ for which GaAs is: $n L>10^{12} \mathrm{~cm}^{-2}$, where $n$ is the electron density and $L$ the device length. It has been argued 
by some that though the inherent carrier concentration in SI-GaAs would not be large to satisfy the Kroemer requirement, photoexcitation would create a high enough electron density to support Gunn domains. However, since higher electron concentrations could be achieved upon photoexcitation, the conventional route of a perturbative field near the cathode route to initiate a domain might not be very likely. In fact, the high density of photoexcited carriers would work to smooth out any perturbative fields arising from imperfections. Separation of the electron-hole pairs due to carrier drift in opposite directions though might help create nonuniform fields internally. This polarization, coupled with the negative differential mobility, could then give rise to enhanced fields within the PCSS. Thus, the possibility of having nonuniform internal electric fields, brought about by charge separation or perhaps through localized charge creation, does remain.

Furthermore, it has been observed that the lock-on behavior is independent of the irradiation intensity as long as this intensity is sufficient to trigger the PCSS into the lockon state. ${ }^{29}$ This observation suggests a minimum carrier density requirement. This aspect is consistent with the qualitative notion of polarization-driven field enhancements, or the need to create a minimum level of carriers to initiate impact ionization.

Double injection has also been proposed as a possible mechanism for lock-on. ${ }^{29}$ However, we take the view that double-injection occurs in semi-insulating GaAs phototoconductive switches, as an independent outcome arising from the large trap densities, coupled with high fields under which such pulsed-power devices operate. Furthermore, though double-injection can lead to instabilities, ${ }^{30}$ we propose this process to be separate from lock-on. Double injection gives rise to S-shaped current-voltage characteristics that promote device instabilities. Filamentary conduction for devices exhibiting S-shaped characteristics was discussed over fifty years ago by Ridley based on thermodynamic considerations. ${ }^{31}$ Two factors, lifetime modulation and internal polarization, lie at the heart of the S-shaped behavior in the context of the SI-GaAs PCSS. The lifetime modulation ${ }^{32,33}$ can simply be understood as follows. In a semi-insulating sample having traps below the Fermi level, the trap levels are mostly occupied by electrons. Hence, the hole lifetime is very short compared to that of the electrons, and conduction is dominated by electronic flow. However, with increasing current (or due to increased photoexcitation), the hole density increases and so does recombination with the filled traps. This then begins to empty the occupied traps. An ever increasing fraction of traps, starting from the cathode side and gradually moving to the anode, thus becomes unoccupied/empty. These unfilled traps can no longer capture holes, and hence the hole lifetime dramatically increases.

A similar modulation of electronic lifetime can also occur if there happens to be empty traps above the Fermi level, which gradually start filling with increasing current. In this scenario, the quasi-Fermi level for electrons simply moves above the trap energy with increasing currents. This would lead to trap filling, and reductions in electron trapping, thereby lengthening the electron lifetimes.
Increases in carrier lifetimes not only enhance currents, but also lead to larger voltage drops across circuit resistances, thus reducing the device potential. Polarization effects, due to charge separation between the dual electron-hole populations, also lower the internal fields. Thus, increased currents with the corresponding device voltage decrease, then leading to an S-shaped characteristic. Since such lifetime modulation depends on the availability of mobile charge to fill traps, it can be facilitated either under large photoexcitation, or when the applied voltage is high enough to force strong carrier injection.

In any event, double-injection is likely to occur in semiconductors with a strong presence of traps, aided by fielddependent processes. ${ }^{34}$ However, the process of doubleinjection does not necessarily require a direct semiconductor material such as GaAs. In fact, the S-shaped negative differential current characteristics have been reported in silicon materials, ${ }^{35}$ without any lock-on. This underscores that filamentation and lock-on are independent effects, although they can both arise in the context of semi-insulating material containing traps.

For completeness, it may be mentioned that strong carrier-carrier scattering has also been suggested as a potential cause for filamentation. ${ }^{36}$ However, carrier-carrier scattering maintains the overall momentum and energy of the system, and these interactions only smooth-out the carrier distribution function. So, dramatic shifts in current, or local heating, or the creation of a large "tail" in the distribution function beyond the ionization threshold is not likely to occur on the basis of carrier-carrier processes.

In this contribution, the time-dependent response of semiinsulating GaAs to laser excitation is studied based on onedimensional (1D) simulations. The focus is on lock-on and the role of the mechanisms that would potentially drive the PCSS into this persistent conductivity mode. The one-dimensional model precludes the analysis of filamentation, though this aspect will be addressed elsewhere. The model solves the continuity equations based on the continuum drift-diffusion theory, including a Poisson solver for self-consistent electric fields and an external circuit series resistance. The inherent physical processes include trap-to-band impact ionization, time- and space-dependent photoexcitation, negative differential resistance of GaAs, both electron and hole injection from the contacts, a trap level with associated emission and capture dynamics (details on the electric field dependence have already been reported by our group ${ }^{37}$ ) and the mechanism of photon recycling. ${ }^{38}$ It is shown that spatially nonuniform electric fields help boost trap-to-band impact ionization and lead to an internal gain mechanism. Furthermore, localized and rapid charge creation due to trap-to-band impact ionization can push the high field region to a neighboring location much like the dynamics of streamer propagation. Hence, fast field movements, multiple domains and charge oscillations, as observed experimentally, can arise.

\section{MODEL DETAILS}

A simple one-dimensional (1D) model, as sketched in Fig. 1, was used for the purposes of modeling the current 
through a biased photoconductive device subject to external illumination. In Fig. 1, the PCSS is in series with a load resistance $\left(R_{L}\right)$ and subject to a voltage supply $\left(V_{O}\right)$. The current flow direction is shown in Fig. 1, and leads to a timedependent device voltage due to the potential drop across the resistor. Calculations of the current were based on the driftdiffusion theory involving the continuity and transport equations given as

$$
\begin{aligned}
d n / d t= & d\left[n(x) v_{n}(F)+D_{n} d n(x) / d x\right] / d x \\
& +e_{\mathrm{n} 0}(F) N_{T i}{ }^{-}-c_{n}(F) n\left(N_{T i}-N_{T i}{ }^{-}\right) \\
& +n N_{T i}{ }^{-} \exp \left[K_{\text {electron }}\left(E_{c}-E_{T i}\right) / F\right] \beta_{n} v_{n} \\
& +K_{1} \sec h^{2}[(t-17) / 2.5] * \exp \left[\alpha\left|z-z_{0}\right|\right] \\
& +K_{2} N_{T}{ }^{-} \sec h^{2}[(t-17) / 2.5] * \exp \left[\alpha\left|z-z_{0}\right|\right],
\end{aligned}
$$

and

$$
\begin{aligned}
d p / d t= & d\left[-p(x) v_{p}(F)+D_{p} d p(x) / d x\right] / d x \\
& +e_{p 0}(F)\left(N_{T i}-N_{T i}{ }^{-}\right)-c_{p}(F) p N_{T i}{ }^{-} \\
& +p\left(N_{T i}-N_{T i}{ }^{-}\right) \exp \left[K_{h o l e}\left(E_{T i}-E_{v}\right) / F\right] \beta_{p} v_{p} \\
& +K_{1} \sec ^{2}[(t-17) / 2.5] * \exp \left[\alpha\left|z-z_{0}\right|\right] \\
& +K_{2}\left(N_{T}-N_{T}{ }^{-}\right) \sec h^{2}[(t-17) / 2.5] * \exp \left[\alpha\left|z-z_{0}\right|\right]
\end{aligned}
$$

where $F$ is the local electric field, $e_{n, p o}$ are the emission rates for electrons and holes, $n, p$ are the electron and hole densities, $v_{n}(F), v_{p}(F)$ are the field-dependent electron and hole drift velocities, $N_{T i}$ and $N_{T i}{ }^{-}$are the total and occupied trap densities at the $i$ th level, $K_{\text {electron }}$ and $K_{\text {hole }}$ are constants for ionization due to electrons and holes, and $\beta_{n, p}$ are equivalent cross-sections for the electron and hole rates. The fourth factor on the right side of Eqs. (1a) and (1b) represents the rate of electron- and hole-assisted trap-to-band impact ionization. The exponential dependence of the trap-to-band ionization coefficients on the electric field was taken to have the same form as derived by Baraff. ${ }^{39}$ Although numerous trap levels

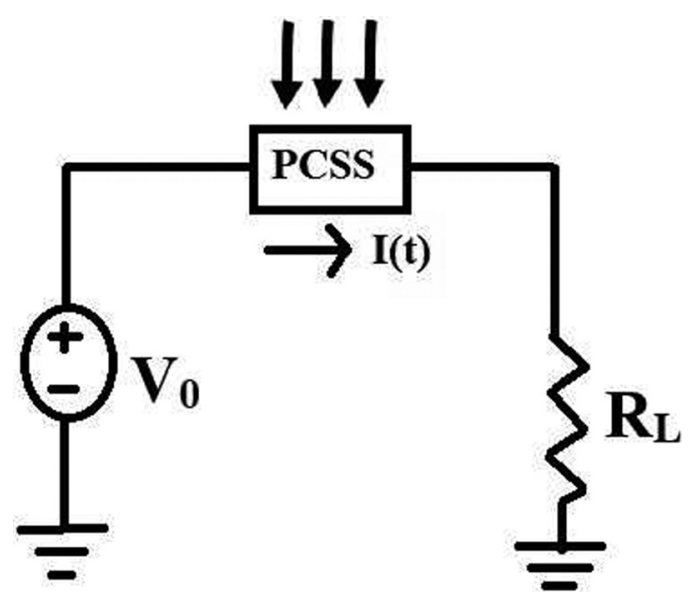

FIG. 1. Schematic of the simulated PCSS showing a constant biasing voltage in series with a load resistor $R_{L}$ and the associated current flow. The illumination incident on the device was assumed to have a hyperbolic secant temporal profile. could have been used, a level $0.75 \mathrm{eV}$ below the conduction band roughly representing the EL2 state was assumed here.

The electric field distribution was updated at every time step from the charge densities based on the Poisson equation as:

$$
d E(x, t) / d x=q\left[p(x, t)+N_{d}(x)-n(x, t)-\sum_{i} N_{T i}{ }^{-}(x, t)\right] / \varepsilon,
$$

where $N_{d}$ represents the background donor density (assumed at a constant value of $7.3 \times 10^{22} \mathrm{~m}^{-3}$ ) and $\varepsilon$ denotes the GaAs permittivity. The boundary conditions for the Poisson solution were taken to be $V_{O}$ and $I(t) R_{L}$ at the two ends, respectively. Here, a nominal $5 \Omega$ series resistance to mimic the external circuit was used. The device current $I(t)$ was calculated at each time step as: $I(t)=(A / N) \sum_{j=1}{ }^{j=N}\left[J_{n}^{j}(t)\right.$ $\left.+J_{p}{ }^{j}(t)\right]+(A \varepsilon / L) d[\Delta V(t)] / d t$, where $N$ denotes the number of grid points along the longitudinal direction, $\Delta V(t)$ is the potential difference across the device, $J_{n, p}{ }^{j}(t)$ represents the calculated electron and hole current densities, respectively, $A$ is the device area, and $L$ denotes the device length. The summation is over the grid points.

The capture rate $c_{n}$ in Eq. (1a) was taken to have a field independent value of $5.1387 \times 10^{3} \mathrm{~m}^{3} \mathrm{~s}^{-1}$ based on a report by Bonilla et al. ${ }^{40}$ The last two factors in (1a) and (1b) represent carrier generation arising from the optical laser pulse due to band-to-band and band-to-trap transitions, respectively. The optical laser pulse was assumed to follow the usual secant-hyperbolic temporal profile. The value of the band-to-band transition parameter was: $K_{l}=1.125 \times 10^{29}$ $\mathrm{m}^{-3} \mathrm{~s}^{-1}$; while the trap-to-band process parameter was taken to be $K_{2}=2.25 \times 10^{7} \mathrm{~s}^{-1}$. Also, in the hyperbolic secant terms of Eq. (1), $t$ represents the time in nanoseconds, $\mathrm{z}$ the position in meters and $\alpha=300 \mathrm{~m}^{-1}$. This yielded a full-width at half maxima of $\sim 2.2 \mathrm{~ns}$, with the peak excitation set to occur at $17 \mathrm{~ns}$ from the start of the simulations, in keeping with the pulse parameters used by Shi et al. ${ }^{41}$ The photon excitation was taken to create mobile conduction electrons from both the valence band and the filled traps. In addition, holes were generated due to the valenceto-conduction band transitions and from excitations between the valence band and unfilled traps. In our model, all concentrations (for both mobile carriers and traps) were taken to be spatially dependent, in a $14 \mathrm{~mm}$ long device with a $140 \mu \mathrm{m}$ grid spacing. The device length was selected in keeping with device sizes used in PCSS experiments reported by Shi et al., ${ }^{41}$ and is typical for high power switching applications. ${ }^{42}$ A cross-sectional area of $2 \mathrm{~cm}^{2}$ was similarly chosen. The time step was $1 \mathrm{ps}$, and satisfied the Courant-Friedrichs-Lewy (CFL) stability criteria. ${ }^{43}$

For GaAs materials, the electron ${ }^{44}$ and hole ${ }^{45}$ drift velocities $\left[v_{n}(F)\right.$ and $v_{p}(F)$, respectively] were taken from the literature. Thus, for the field-dependent electron drift velocity $v_{n}(F)$

$$
\begin{aligned}
v_{n}(F \geq 0)= & {\left[\mu_{0} F+v_{\text {sat }}\left(F / F_{t h}\right)^{4}\right] /\left[1+\left(F / F_{t h}\right)^{4}\right], } \\
& \text { and } v_{n}(F<0)=-v_{n}(F>0),
\end{aligned}
$$


where $\mu_{0}=5800 \mathrm{~cm}^{2} /(\mathrm{Vs}), v_{\text {sat }}=7.5 \times 10^{6} \mathrm{~cm} / \mathrm{s}, F_{\text {th }}=4 \mathrm{kV} /$ $\mathrm{cm}$, and $F$ denotes the electric field. For the field-dependent hole drift velocity $v_{p}(F)$, the following relation was used: ${ }^{45}$

$$
\begin{aligned}
v_{p}(F \geq 0)= & 5.9 \times 10^{3} z_{h}{ }^{7}-3.1 \times 10^{4} z_{h}{ }^{6}+5.6 \\
& \times 10^{4} z_{h}{ }^{5}-3.5 \times 10^{4} z_{h}{ }^{4}-6 \times 10^{3} z_{h}{ }^{3} \\
& +1.3 \times 10^{4} z_{h}{ }^{2}-2.8 \times 10^{3} z_{h}+7 \times 10^{4},
\end{aligned}
$$

with $z_{h}=\left(|F|-2.3 \times 10^{7}\right) /\left(2.5 \times 10^{7}\right), \quad$ and $v_{p}(F<0)=-v_{p}(F>0)$.

The possibility of hole injection from the anode due to electrons collected at the contact was also included. Electrons exiting the GaAs sample and incident on the anode contact could form holes by transferring energy to other electrons in the anode and raising their energy above the Fermi level. This would leave holes below the Fermi sea leading to their injection into GaAs. Here, for simplicity, the injected hole flux was assigned to be some fraction $\left(R_{\text {inj }}(F)\right)$ of the electron inflow. Thus, $J_{p}=J_{n} R_{i n j}(F)$, with $J_{p}$ and $J_{n}$ denoting the hole and electron current densities and $R_{i n j}(F)$ the hole-injection probability factor taken to be an adjustable parameter.

Finally, photon re-cycling aspects were included and are briefly discussed for completeness, with details given in the Appendix. Consider first transitions involving traps and the occupied conduction band. Photons generated due to radiative recombination at any position $z^{\prime}$ can move to a different location $z$, while being attenuated during their traversal, and then be re-absorbed. This re-absorption due to the recycled photons reaching location $z$ will induce transitions from filled traps to the conduction band. Thus, the electron density at location $z$ would increase, while the density of occupied traps $N_{T}^{-}(z)$ would reduce, leading to

$$
n_{\text {new }}(z)=n(z)+G_{\mathrm{ph}}(z) N_{T}{ }^{-}(z) \mathrm{dt},
$$

and

$$
N_{T}{ }^{-}{ }_{\text {new }}(z)=N_{T}{ }^{-}(z)-G_{p h}(z) N_{T}{ }^{-}(z) d t
$$

In the above, $G_{p h}(z)$ represents the photon generation rate at location $z$ as given in the Appendix, while $n_{\text {new }}(z)$ and $N_{T}^{-}{ }_{n e w}(z)$ denote the updated electron and occupied trap densities. Similarly, one needs to account for changes in densities at initial locations $z^{\prime}$ that are responsible for photon creation in the first place. The photon creation processes reduce the density $n\left(z^{\prime}\right)$ and increase $N_{T}^{-}\left(z^{\prime}\right)$. Taking these processes into account, one gets (as shown in the Appendix)

$$
\begin{aligned}
n_{\text {new }}\left(z^{\prime}\right)= & n\left(z^{\prime}\right)-C^{*}\left[n\left(z^{\prime}\right) / N_{c}\right] \\
& \times\left\{\left[N_{T}\left(z^{\prime}\right)-N_{T}-\left(z^{\prime}\right)\right] / N_{T}\right\} d t,
\end{aligned}
$$

and

$$
\begin{aligned}
N_{T}{ }_{\text {new }}\left(z^{\prime}\right)= & N_{T}{ }^{-}\left(z^{\prime}\right)+C^{*}\left[n\left(z^{\prime}\right) / N_{c}\right] \\
& \times\left\{\left[N_{T}\left(z^{\prime}\right)-N_{T}{ }^{-}\left(z^{\prime}\right)\right] / N_{T}\right\} d t .
\end{aligned}
$$

Similar considerations apply for transitions involving occupied traps and holes in the valence band at location $z^{\prime}$ and lead to the following time-dependent changes in the holes and occupied trap levels:

$$
\begin{aligned}
p_{\text {new }}\left(\mathrm{z}^{\prime}\right)=\mathrm{p}\left(\mathrm{z}^{\prime}\right)-C^{*}[ & \left.p\left(\mathrm{z}^{\prime}\right) / N_{v}\right]\left[N_{T}{ }^{-}\left(\mathrm{z}^{\prime}\right) / N_{T}\right] \mathrm{dt}, \\
\text { and, } N_{T}{ }^{-}{ }_{\text {new }}\left(z^{\prime}\right)= & N_{T}{ }^{-}\left(z^{\prime}\right)-C^{*}\left[p\left(z^{\prime}\right) / N_{v}\right] \\
& \times\left[N_{T}{ }^{-}\left(z^{\prime}\right) / N_{T}\right] d t .
\end{aligned}
$$

Finally, for the transitions between empty traps and valence electrons at location $z$ involving re-absorbed photons, the relations are

$$
p_{\text {new }}(z)=p(z)+G_{p h}(z)\left[N_{T}-N_{T}^{-}(z)\right] d t,
$$

and

$$
N_{T}{ }^{-}{ }_{n e w}(z)=N_{T}{ }^{-}(z)+G_{p h}(z)\left[N_{T}-N_{T}{ }^{-}(z)\right] d t .
$$

\section{RESULTS AND DISCUSSION}

\section{A. Time dependent currents}

One-dimensional, time-dependent simulations for the GaAs PCSS, based on the model described above, were performed. The parameters used are given in Table I. Although many simulations under a variety of different cases were carried out, only the important results have been shown and are

\begin{tabular}{|c|c|}
\hline Parameter & Value \\
\hline Hole injection ratio, $R_{i n j}$ & 0.02 \\
\hline $\begin{array}{l}\text { Parameter } C_{1} \text { for electrons due to photon } \\
\text { recycled absorption }\end{array}$ & $2.596 \mathrm{~s}^{-1}$ \\
\hline $\begin{array}{l}\text { Parameter } C_{1} \text { for holes due to photon } \\
\text { recycled absorption }\end{array}$ & $2.36 \mathrm{~s}^{-1}$ \\
\hline $\begin{array}{l}\text { Parameter } C^{*} \text { for electrons due } \\
\text { to photon generation }\end{array}$ & $1.2876 \times 10^{-5} \mathrm{~s}^{-1}$ \\
\hline $\begin{array}{l}\text { Parameter } C^{*} \text { for holes due to photon } \\
\text { generation }\end{array}$ & $1.4164 \times 10^{-5} \mathrm{~s}^{-1}$ \\
\hline Photon absorption co-efficient & $7 \times 10^{5} \mathrm{~m}^{-1}$ \\
\hline $\begin{array}{l}\text { Electric field and energy gap dependence } \\
\text { factor for electrons, } K_{\text {electron }}\end{array}$ & $72 \mathrm{kV} /(\mathrm{eV} \mathrm{cm})$ \\
\hline $\begin{array}{l}\text { Electric field and energy gap dependence } \\
\text { factor for holes, } K_{\text {holes }}\end{array}$ & $64 \mathrm{kV} /(\mathrm{eV} \mathrm{cm})$ \\
\hline Equivalent cross-section for electrons, $\beta_{n}$ & $8.07 \times 10^{-16} \mathrm{~m}^{2}$ \\
\hline Equivalent cross-section for holes, $\beta_{p}$ & $4.81 \times 10^{-16} \mathrm{~m}^{2}$ \\
\hline
\end{tabular}
discussed here. The role of the average electric field across the $14 \mathrm{~mm}$ PCSS device was probed first. For these simulations, the laser pulse was centered at the $17 \mathrm{~ns}$ instant (as already described), and was taken to have a spatially symmetric profile with peak intensity located at the midway point. Time dependent PCSS current results at two different average electric fields of $3 \mathrm{kV} / \mathrm{cm}$ and $7.14 \mathrm{kV} / \mathrm{cm}$ are shown in Fig. 2. As evident from the curves, current decay is predicted at the lower field, while a persistent current with fluctuations is seen at the higher field. In fact, from the various simulations performed (but not shown for brevity), an average field value of at least $3.5 \mathrm{kV} / \mathrm{cm}$ was seen to be necessary

TABLE I. Parameters used for the time dependent GaAs simulations. 


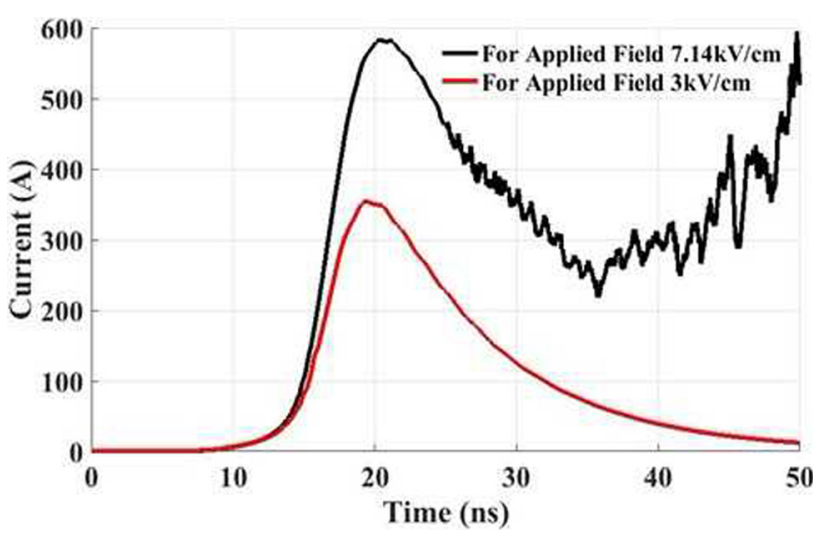

FIG. 2. Simulation results for the time dependent current response of the PCSS at two different average electric fields of $3 \mathrm{kV} / \mathrm{cm}$ and $7.14 \mathrm{kV} / \mathrm{cm}$. Current decay is predicted at the lower field, while a persistent current with fluctuations is seen at the higher field.

to avoid current decays. This aspect is roughly in keeping with the experimental observations of a minimum threshold around $4 \mathrm{kV} / \mathrm{cm}$ for lock-on in semi-insulating GaAs photoconductive switches.

Next, comparisons between the simulation results for the PCSS current response at the $7.14 \mathrm{kV} / \mathrm{cm}$ average field with actual data obtained by Shi et al. ${ }^{41}$ were made. The results are shown in Fig. 3. The current magnitudes are similar, and fluctuations are seen to occur for both curves. This is an important point and indicative of fast internal dynamic changes that will be discussed in detail later. The general trend and the current magnitudes obtained here lend validity to the model developed.

From the standpoint of probing the inherent physics and the relative role of the underlying processes, simulation runs were carried out for a set of different conditions. The time dependent PCSS currents at the $7.14 \mathrm{kV} / \mathrm{cm}$ average field were obtained for three cases: (i) inclusion of both trap-toband impact ionization and photon recycling, (ii) presence of only the impact ionization without any photon recycling, and (iii) a simple version with neither impact ionization, nor photon recycling, taken into account. The results are shown in Fig. 4, and in these simulations, the peak values of the

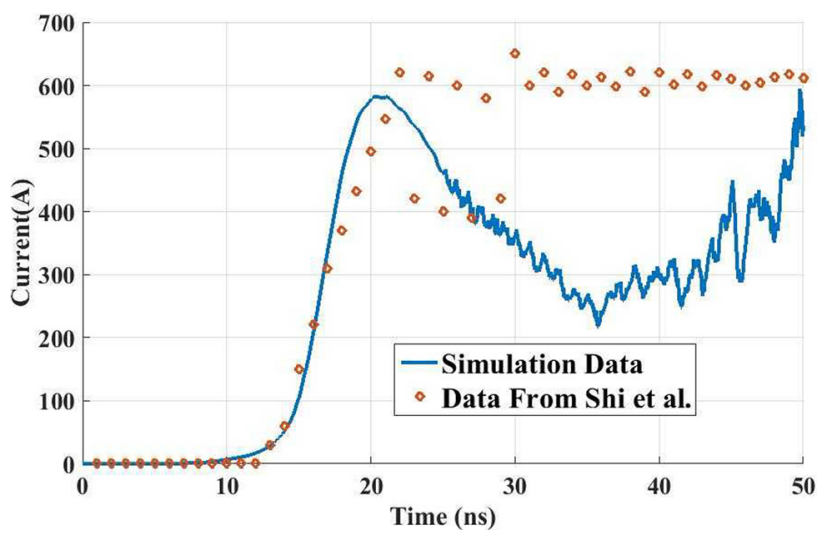

FIG. 3. Comparison between simulation results for the time dependent PCSS current response at the $7.14 \mathrm{kV} / \mathrm{cm}$ average field with data obtained by Shi et al. ${ }^{41}$ The magnitudes are comparable and fluctuations are seen to occur in both cases. generation rates for band-to-trap and band-to-band were chosen to be $2.25 \times 10^{7} \mathrm{~s}^{-1}$ and $1.125 \times 10^{29} \mathrm{~m}^{-3} \mathrm{~s}^{-1}$, respectively. The largest current with a clear persistent pattern is seen to occur only when both impact ionization and photon recycling were included. The values in the 450-500 A range are predicted, with some fluctuations occurring once the current becomes fairly large. When neither of the two processes was taken into account, the photo-current was short-lived, stayed below $50 \mathrm{~A}$, and was predicted to decay rapidly while roughly following the optical waveform. With photon recycling included, but without impact ionization, the current in Fig. 4 is also seen to decay, though it lasts for a longer time of about 30 ns.

The collective picture that then emerges from these results is the dominant role played by trap-to-band impact ionization in helping to sustain the photocurrent and bringing the PCSS into the lock-on mode. Furthermore, though photon recycling appears to have a positive role in enhancing the current, it does not by itself take the PCSS into a lock-on state. It does, however, point to the benefits of better photon confinement within the photoconductor. Finally, under lockon conditions, the results are indicative of fairly rapid fluctuations. These, as explained subsequently, arise from the repeated initiation of internal charge generation at different sites within the PCSS due to the presence of high local fields.

In addition, a lower level of laser generation was found inadequate to support lock-on via our simulations. This can be seen from the simulation result of Fig. 5. The curves in this plot were obtained for two different laser generation values. At a reduced generation level, the peak current value is predicted to be lower ( $\sim 400 \mathrm{~A}$ ) as compared to the $\sim 600 \mathrm{~A}$ with a higher generation rate. Also, for the lower generation rate, the current gradually decreases over time. Though not specifically shown here for brevity, with lower degrees of photon recycling, a higher applied voltage was required to drive the PCSS into lock-on, in keeping with experimental reports in the literature.

\section{B. Spatial profiles}

Having probed the external current and its timedependent behavior, the spatio-temporal evolution of the

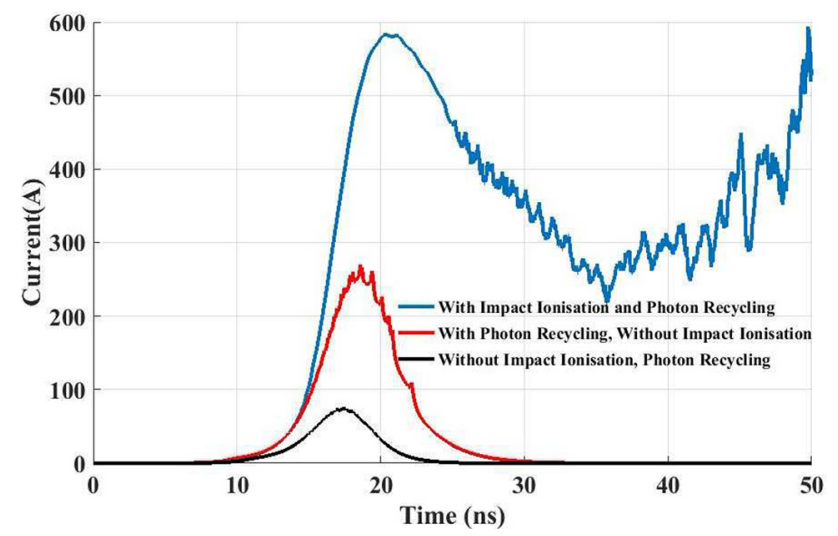

FIG. 4. Results obtained for the time dependent PCSS current at the $7.14 \mathrm{kV} / \mathrm{cm}$ average field for three different simulation cases. 


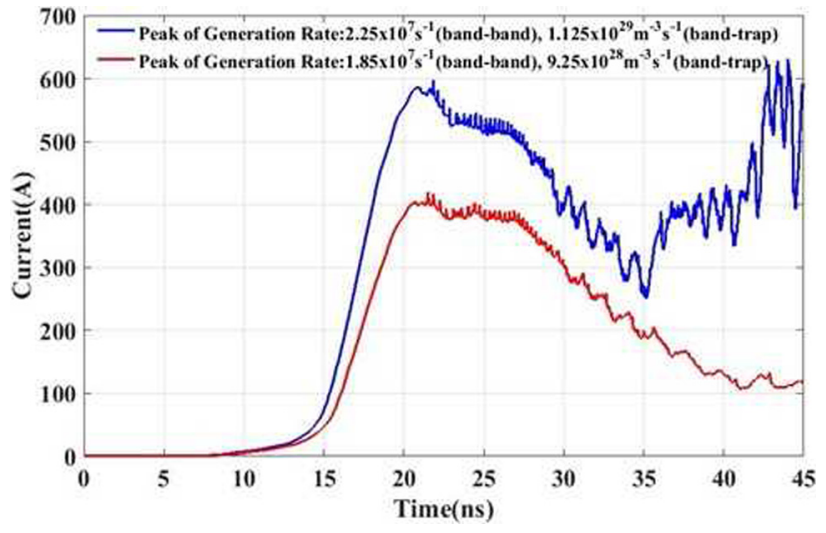

FIG. 5. Results of the time dependent PCSS current at the $7.14 \mathrm{kV} / \mathrm{cm}$ average field obtained with impact ionization and two different generation rates.

internal electric fields and charge distributions that produce the current, was analyzed next. Figure 6 shows the 10 ns, 11 ns, and 12 ns snapshots of the electric field and electron density profiles within the PCSS. The laser was spatially focused at the center of the device with an exponential fall-off from the central position. The results were obtained for the $7.14 \mathrm{kV} / \mathrm{cm}$ average field case with inclusion of both impact ionization and photon recycling. The electron density [as shown in Fig. 6(b)] has the highest values at the device center. This supports a strong conduction current component, but works to reduce the displacement currents, to satisfy total current continuity. Consequently, electric fields reduce over the region of enhanced carrier densities. Figure 6(a) reflects this, with electric fields falling over time and also spreading out from the center spot. The relatively high field regions then develop over the two end regions. This also implies that conditions for initiation of impact ionization would be created at the two end zones.

Such dynamic changes continue and snapshots of the electric field and electron density profiles obtained at the 27 ns, $28 \mathrm{~ns}$ and $29 \mathrm{~ns}$ time instants are shown in Fig. 7. Two important aspects seen in these results are: (i) the appearance of high electric field regions towards the cathode (on the left) and anode (on right) sides of the PCSS and (ii) the movement of these high field regions towards each other is at a speed of
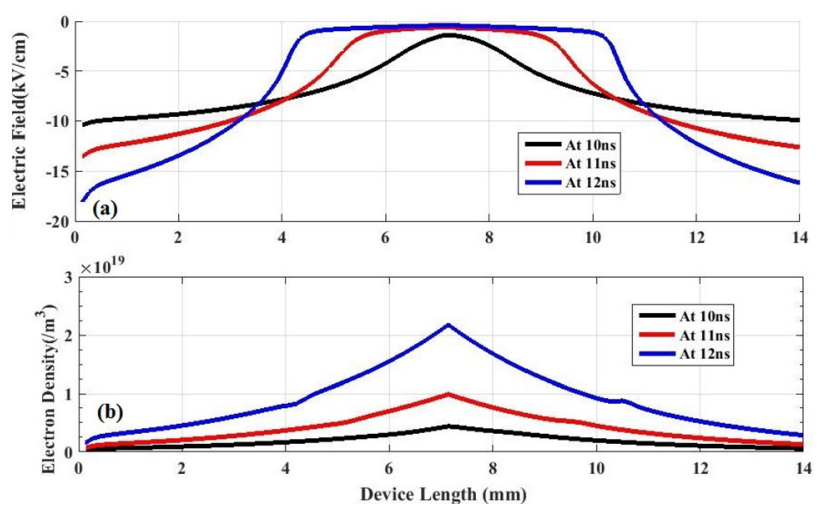

FIG. 6. Snapshots at $10 \mathrm{~ns}, 11 \mathrm{~ns}$ and $12 \mathrm{~ns}$ for the $7.14 \mathrm{kV} / \mathrm{cm}$ average field case with inclusion of both impact ionization and photon recycling. The laser was spatially focused at the center of the device. (a) Temporal evolution of the electric field profile and (b) the electron density profile.
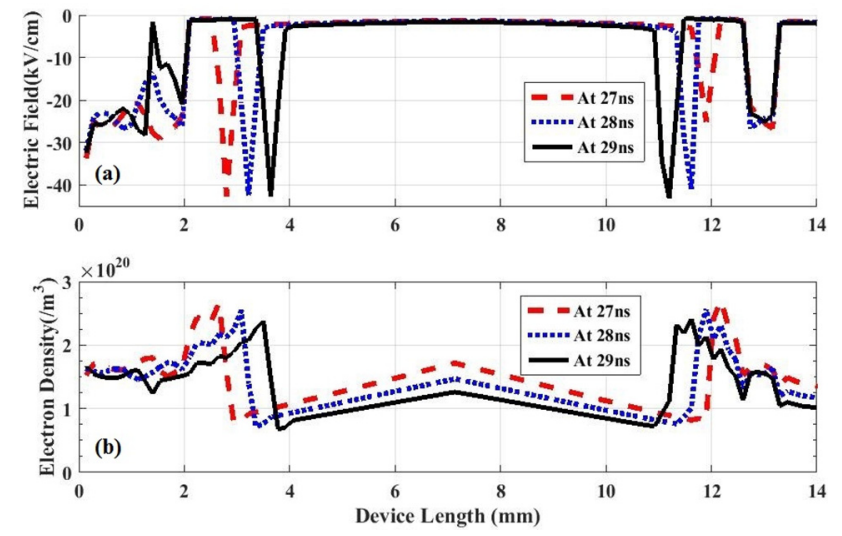

FIG. 7. Snapshots at $27 \mathrm{~ns}, 28 \mathrm{~ns}$ and $29 \mathrm{~ns}$ for the $7.14 \mathrm{kV} / \mathrm{cm}$ average field case with inclusion of both impact ionization and photon recycling. The laser was spatially focused at the center of the device. (a) Temporal evolution of the electric field profile and (b) the electron density profile.

$\sim 9 \times 10^{5} \mathrm{~m} / \mathrm{s}$. These speeds are much higher than the highest electron drift velocity in GaAs. This can be understood as follows: (a) there are initial electron density increases due to photoexcitation. (b) The ensuing local electric fields then decrease over these regions of high carrier density, followed by a progression of the electric field to an adjacent region much like the movement of a streamer. (c) With a shift in the electric field to an adjacent location, strong enhancements in electron density through trap-to-band impact ionization then develop, and a positive feedback cycle with a moving charge domain is initiated.

The electric field movement can be seen from the results of Fig. 7(a). Consider the electric field peak on the left at the $27 \mathrm{~ns}$ instant. The electron density just to the left, as seen in Fig. 7(b), is higher than the value to the right of the peak field. Consequently, over time, the peak field advances to the right (where the conduction currents were low and required a higher displacement component) from about $2.75 \mathrm{~mm}$ to the $3.65 \mathrm{~mm}$ location, during the $27 \mathrm{~ns}$ to $28 \mathrm{~ns}$ time interval. Similarly, for the electric field peak on the right located at about $12 \mathrm{~mm}$, the movement is to the left since the electron density exhibits higher values to the right of the $12 \mathrm{~mm}$ location.

The progressive movement of the localized electric field peaks on the left and right sides towards each other is even more apparent in Figs. 8(a) and 8(b), which are the electric field and electron density snapshots, respectively, at the 29 ns, $37 \mathrm{~ns}$, and $45 \mathrm{~ns}$ instants. An important development, that of multiple-domain formation, is predicted at longer times in Figs. 8(a) and 8(b). The 29 ns snapshot reveals only two primary high field regions on the left and right sides in Fig. 8(a). However, at $37 \mathrm{~ns}$, these two primary field zones are seen to have merged roughly at the $7.7 \mathrm{~mm}$ location, with the creation of two more high-field side zones at about the $1.7 \mathrm{~mm}$ and $12.8 \mathrm{~mm}$ positions. Very crudely, the combination of the two primary high-field zones redistributes the potential. The voltage reduction upon coalescence is compensated through the emergence of two new high-field regions. The net multi-domain structure provides for a distributed, but enhanced source of charge creation via trap-toband ionization. The cumulative charge density throughout 

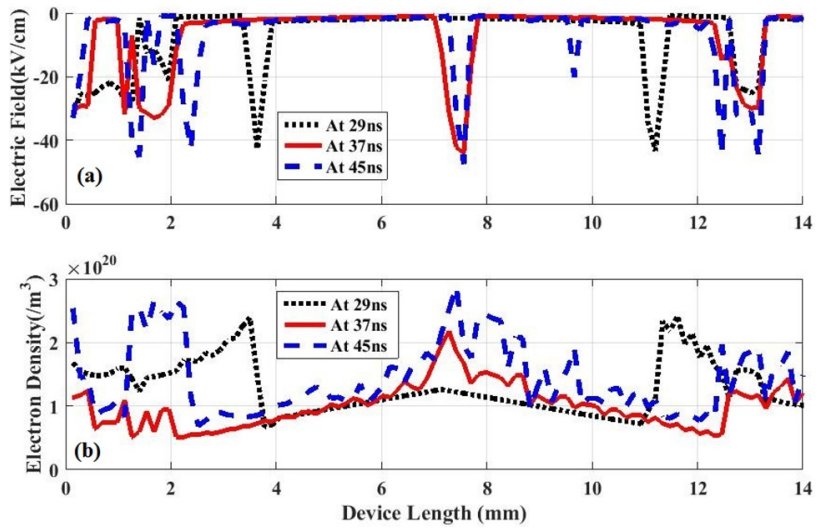

FIG. 8. Snapshots at $29 \mathrm{~ns}, 37 \mathrm{~ns}$ and $45 \mathrm{~ns}$ for the $7.14 \mathrm{kV} / \mathrm{cm}$ average field case with inclusion of both impact ionization and photon recycling. The laser was spatially focused at the center of the device. (a) Temporal evolution of the electric field profile and (b) the electron density profile.

the device is also higher at $45 \mathrm{~ns}$. Though this aspect may not easily be seen in Fig. 8(b), the tangible increase in external current at $45 \mathrm{~ns}$ as compared to the $37 \mathrm{~ns}$ instant is obvious in Fig. 2.

Incidentally, the possibility of multiple domains that would help sustain high device currents has not been discussed much in the literature. The first report of multiple domains with potential for oscillatory instability by Kroemer ${ }^{46}$ was in the context of a steep voltage front (large $d V / d t$ ) applied to a semiconductor. A similar scenario of multiple domains was discussed in the context of bipolar GaAs transistors by Vainshtein et al ${ }^{25}$ In our case, fluctuations in carrier density collectively caused by the spatial variation in photogeneration, the consequent creation of nonuniform fields, the field-dependent velocities, and nonlinear dependency of carrier impact generation on both the density and the electric field are responsible for producing multiple domains. The possibility of multiple domains due to fluctuations in doping density was already suggested by Thim. ${ }^{22}$ Furthermore, the build-up of fields within the device followed by their quenching should produce two effects: (i) continuous localized sources for current growth at different sites and (ii) fluctuations in current due to the spontaneous formation followed by quenching of the high electric fields.

\section{Location of laser excitation}

For completeness, the aspect of position dependent triggering of a PCSS is also discussed. Some studies seem to suggest that laser triggering at locations nearer the cathode would create larger on-state switch conductances and greater currents, or require smaller applied voltages for a given current level. ${ }^{47,48}$ To probe these aspects further, the present model was applied to simulate three different cases with the laser profile centered at $25 \%, 50 \%$ and $75 \%$ of the device length. This corresponds to distances of $3.5 \mathrm{~mm}, 7.5 \mathrm{~mm}$ and $10.5 \mathrm{~mm}$ from the left (cathode) end. Both impact ionization and photon recycling were included. The results obtained are shown in Fig. 9. Of the three cases, the highest currents are predicted with the laser nearer the cathode at $25 \%$ of the device length. The symmetrically centered profile (with the

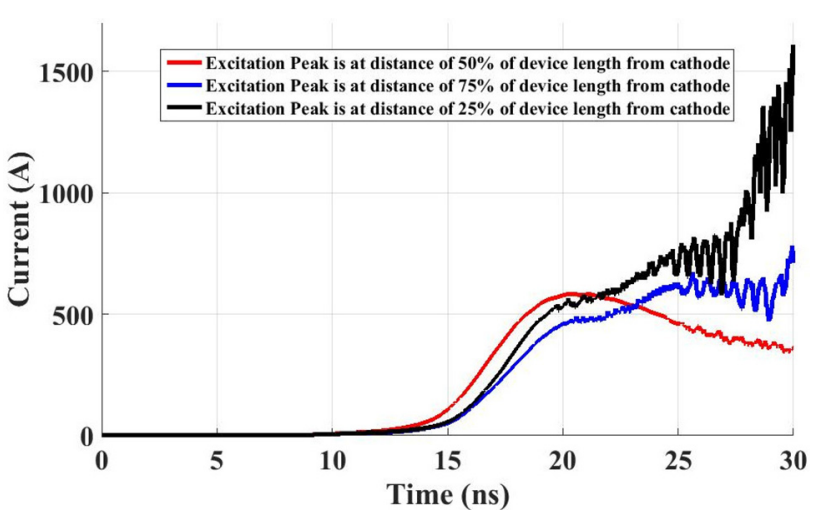

FIG. 9. Results of the time dependent PCSS current at the $7.14 \mathrm{kV} / \mathrm{cm}$ average field obtained for three different locations of the laser focal point. $25 \%$, $50 \%$ and $75 \%$ of the device length represent the laser centered at distances of $3.5 \mathrm{~mm}, 7.5 \mathrm{~mm}$ and $10.5 \mathrm{~mm}$ from the left (cathode) end. Both impact ionization and photon recycling were included.

peak at $50 \%$ of the device length) had the smallest current, while the profile near the anode is at three-fourths the distance having an intermediate response.

Figures 10(a) and 10(b) show snapshots of the electric field and electron density profiles obtained at $18 \mathrm{~ns}, 19 \mathrm{~ns}, 20$ $\mathrm{ns}$, and $22 \mathrm{~ns}$ for the $7.14 \mathrm{kV} / \mathrm{cm}$ average field case, with laser centered $3.5 \mathrm{~mm}$ from the cathode on the left. As might be expected, since the carrier creation predominantly occurs on the left, the high field regions are formed on the right side. Multiple field domains are predicted to develop in Fig. 10 (a) at the longest $22 \mathrm{~ns}$ time. Furthermore, both the high electric field region and the carrier density peaks move to the cathode on the left. Also, the fluctuations in Fig. 9 roughly start at $22 \mathrm{~ns}$ time, when the multiple domains appear to be initiated in Fig. 10(a). Finally, Figs. 11(a) and 11(b) show the temporal evolution of the spatial profiles for the case of the laser centered at the $10.5 \mathrm{~mm}$ location from the left at $75 \%$ of the device length. The behavior is almost a mirror reflection of Figs. 10(a) and 10(b). The highest peak field in Fig. 11(a) is not quite as large as that in Fig. 10(a). This is probably the result of electron injection from the cathode.
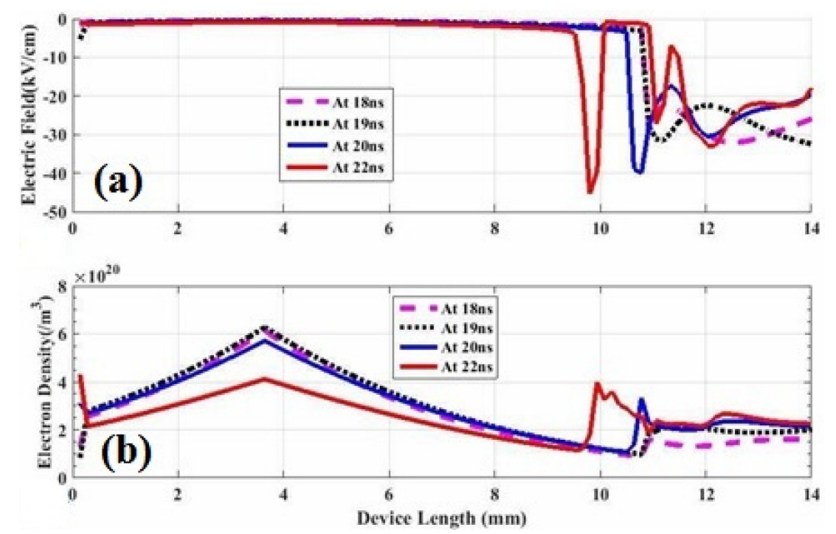

FIG. 10. Snapshots at $18 \mathrm{~ns}, 19 \mathrm{~ns}, 20 \mathrm{~ns}$, and $22 \mathrm{~ns}$ for the $7.14 \mathrm{kV} / \mathrm{cm}$ average field case, with laser centered at a spot a distance of $3.5 \mathrm{~mm}$ away from the cathode on the left. Both impact ionization and photon recycling were included. (a) Temporal evolution of the electric field profile and (b) the electron density profile. 

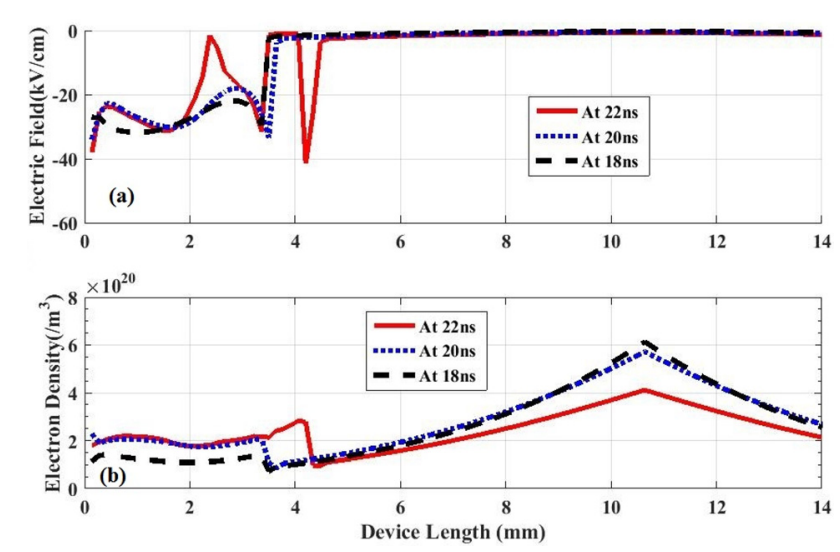

FIG. 11. Snapshots at $18 \mathrm{~ns}, 20 \mathrm{~ns}$, and $22 \mathrm{~ns}$ for the $7.14 \mathrm{kV} / \mathrm{cm}$ average field case, with laser centered at a spot a distance of $3.5 \mathrm{~mm}$ away from the anode on the right. Both impact ionization and photon recycling were included. (a) Temporal evolution of the electric field profile and (b) the electron density profile.

\section{SUMMARIZING CONCLUSIONS}

The time-dependent response of semi-insulating GaAs to laser excitation was studied based on one-dimensional, time-dependent simulations. The focus was to model the lock-on phenomenon, with quantitative evaluations into the role of various mechanisms that could potentially drive the PCSS into this persistent conductivity mode. The model solved the continuity equations based on the continuum drift-diffusion theory, including a Poisson solver for self-consistent electric fields and a circuit resistor for external effects. Our treatment also included trap-toband impact ionization, time- and space-dependent photoexcitation, negative differential resistance of GaAs, injection from the contacts, and the mechanism of photon recycling.

The numerical results show that spatially nonuniform electric fields can be created by the spatially nonuniform laser excitation, space-charge separation, and the intervalley transfers in GaAs. The local field enhancements help boost trap-to-band impact ionization, which then furnishes a supply of mobile charge to help sustain currents. Our results also suggest that photon recycling is a contributing factor in GaAs. Predictions were indicative of a persistent current above an average field of $3.5 \mathrm{kV} / \mathrm{cm}$. This result is in keeping with experimental observations of a threshold lock-on value of around $4 \mathrm{kV} / \mathrm{cm}^{13}$ In addition, the simulation results obtained compared well with actual data obtained by Shi et $a l .{ }^{41}$ These values obtained were also in the same range as reported by the Sandia group. ${ }^{13}$

The temporal photocurrent response was seen to depend on the illumination intensity. Persistent current for an adequately large electric field only resulted when the photogeneration rate was strong. Such a dependence on illumination intensity is also in keeping with observations. Finally, our simulations showed the possibility of moving multiple domains with potential for oscillatory instability at high voltages. The possibility of filamentation can then arise. However, such an analysis will require two-dimensional simulations, and will be reported elsewhere.

\section{ACKNOWLEDGMENTS}

Useful discussions with G. Laity (Sandia National Laboratories) are greatly appreciated.

\section{APPENDIX: DERIVATION OF PHOTON RECYCLING EQUATIONS FOR ELECTRONS AND HOLES}

Considering a one-dimensional structure of length $L_{z}$ along the $z$-direction, photons generated at any position $z^{\prime}$ can reach a different location $z$ and be re-absorbed, while being attenuated during the traversal process. The generation rate of such photons at the point of origination $z^{\prime}$ is proportional to $n\left(z^{\prime}, t\right) / \tau$, with $\tau$ being the radiative recombination time constant and $n\left(z^{\prime}, t\right)$ the electron density at $z^{\prime}$. Assuming a 50-50 split between the forward and backward propagation of the emitted photons, and an attenuation factor of $\exp \left(-\alpha\left|z^{\prime}-z^{\prime}\right|\right)$, implies that the photon intensity at the re-absorption point would then scale as: $0.5\left[n\left(z^{\prime}, t\right) / \tau\right]$ $\exp \left(-\alpha\left|z^{\prime}-z^{\prime}\right|\right)$. Taking into account that the actual absorption of the photon flux is proportional to the absorption coefficient $\alpha(h \nu)$, the electron generation rate per unit volume at the location is

$G_{p h} \sim \int_{0}^{L z} \int_{0}^{\infty} \alpha(h \nu)\left\{n\left(z^{\prime}\right) / \tau\right\}\left(\Upsilon_{s p} / 2\right) \exp \left[-\alpha\left|z-z^{\prime}\right|\right] d z^{\prime} d(h \nu)$,

where $\Upsilon_{s p}$ is the spontaneous generation rate of photons, and the factor $1 / 2$ takes account of the 50-50 isotropic split of the radiated photons.

Based on the light emission produced by electronic transitions from the conduction band to traps, one has

$$
\begin{gathered}
\Upsilon_{s p}(h \nu) \sim K_{1} \gamma_{\text {spon }}(h \nu)\left[N_{T}\left(z^{\prime}\right)-N_{T}{ }^{-}\left(z^{\prime}\right)\right] \\
\text { where, } \int \gamma_{\text {spon }}(\mathrm{h} \nu) d(\mathrm{~h} \nu)=1
\end{gathered}
$$

and $\gamma_{\text {spon }}(h \nu)$ denotes the normalized emission spectrum. However, for completeness, one has to recognize that emissions arise from the transition into trap states and a distribution of energies exists in the conduction band. Consequently: $n(z) \sim K_{E c} \int^{\infty}\left(E-E_{c}\right)^{1 / 2} \exp \left[\left(E_{F n}-E\right) /(k T)\right]$ $d E=N_{c} \exp \left[\left(E_{F n}-E_{C}\right) /(k T)\right]$, where $E_{F n}$ is the electron quasi-Fermi level and $h \nu=\left(E-E_{T}\right)$ with the lowest frequency $\nu_{\text {low }}$ being: $\nu_{\text {low }}=\left(E_{C}-E_{T}\right) / h$. One obtains from Eqs. (A1), (A2a), and (A2b)

$$
\begin{aligned}
G_{p h} \sim & K^{*} \int_{0}^{L z} \nu_{\text {low }} \int^{\infty} \alpha(h \nu)\left\{\gamma_{\text {spon }}(h \nu) / \tau\right\} \\
& \times\left[N_{T}\left(z^{\prime}\right)-N_{T}^{-}\left(z^{\prime}\right)\right]\left(h \nu+E_{T}-E_{c}\right)^{1 / 2} \\
& \times \exp \left[\left(E_{F n}-h \nu-E_{T}\right) /(k T)\right] \\
& \times \exp \left[-\alpha\left|z-z^{\prime}\right|\right] d z^{\prime} d(h \nu)
\end{aligned}
$$

Using: $\exp \left[\left(E_{F n}-h \nu-E_{T}\right) /(k T)\right]=\exp \left[\left(E_{F n}-E_{C}\right) /(k T)\right]$ $\times \exp \left[\left(E_{C}-h \nu-E_{T}\right) /(k T)\right]=\left[n / N_{c}\right] \exp \left[\left(E_{C}-h \nu-E_{T}\right) /\right.$ $(k T)]$, some straight-forward algebra yields 


$$
\begin{aligned}
G_{p h} \sim & K_{0} \int^{L z} \nu_{\text {low }} \int^{\infty} \alpha(h \nu)\left\{\gamma_{\text {spon }}(h \nu) / \tau\right\} \\
& \times\left[N_{T}\left(z^{\prime}\right)-N_{T}^{-}\left(z^{\prime}\right)\right]\left(h \nu+E_{T}-E_{c}\right)^{1 / 2} \\
& \times\left[n\left(z^{\prime}\right) / N_{c}\right] \exp \left[\left(E_{C}-h \nu-E_{T}\right) /(k T)\right] \\
& \times \exp \left[-\alpha\left|z-z^{\prime}\right|\right] d z^{\prime} d(h \nu), \\
\text { i.e., } G_{p h} \sim & \left(K^{*} N_{T} / N_{c}\right) \int_{0}^{L z} \nu_{l o w} \int^{\infty} \alpha(h \nu) \\
& \times\left\{\gamma_{s p o n}(h \nu) / \tau\right\}\left\{\left[N_{T}\left(z^{\prime}\right)-N_{T}{ }^{-}\left(z^{\prime}\right)\right] / N_{T}\right\} \\
& \times\left(h \nu+E_{T}-E_{c}\right)^{1 / 2}\left[n\left(z^{\prime}\right)\right] \\
& \times \exp \left[\left(E_{C}-h \nu-E_{T}\right) /(k T)\right] \\
& \times \exp \left[-\alpha\left|z-z^{\prime}\right|\right] d z^{\prime} d(h \nu) .
\end{aligned}
$$

This can finally be expressed as

$$
G_{p h} \sim C_{1} \int_{0}^{L z}\left\{\left[N_{T}\left(z^{\prime}\right)-N_{T}^{-}\left(z^{\prime}\right)\right] / N_{T}\right\}\left[n\left(z^{\prime}\right)\right] d z^{\prime}
$$

The absorption of recycled photons reaching location $z$ will increase the electron density at location $z$, while reducing the density of occupied traps $N_{T}^{-}(z)$ as

$$
n_{\text {new }}(z)=n(z)+G_{p h}(z) N_{T}{ }^{-}(z) d t,
$$

and

$$
N_{T}{ }^{-}{ }_{n e w}(z)=N_{T}{ }^{-}(z)-G_{p h}(z) N_{T}{ }^{-}(z) d t .
$$

The above expression (A5a) yields the additional electron density created in the conduction band due to photon recycling.

One also needs to account for changes in the density at the initial locations $z^{\prime}$ responsible for the creation of photons in the first place. These processes reduce the density $n\left(z^{\prime}\right)$ and increase $N_{T}^{-}\left(z^{\prime}\right)$, yielding

$$
\begin{aligned}
n_{\text {new }}\left(z^{\prime}\right)= & n\left(z^{\prime}\right)-K^{*} n\left(z^{\prime}\right)\left[N_{T}\left(z^{\prime}\right)-N_{T}{ }^{-}\left(z^{\prime}\right)\right]_{(E C-E T) / h} \\
& \times \int^{\infty} \alpha(h \nu) \nu^{2}\left(h \nu+E_{T}-E_{c}\right)^{1 / 2} \\
& \times \exp \left[\left(E_{C}-h \nu-E_{T}\right) /(k T)\right] d(h \nu) d t .
\end{aligned}
$$

(A6a)

The above can then be reworked and expressed in the following form, along with a corresponding expression for $N_{T \text { new }}^{-}\left(z^{\prime}\right)$ as

$$
\begin{aligned}
n_{\text {new }}\left(z^{\prime}\right)= & n\left(z^{\prime}\right)-C^{*}\left[n\left(z^{\prime}\right) / N_{c}\right] \\
& \times\left\{\left[N_{T}\left(z^{\prime}\right)-N_{T}^{-}\left(z^{\prime}\right)\right] / N_{T}\right\} d t
\end{aligned}
$$

and

$$
\begin{aligned}
N_{T}{ }_{\text {new }}\left(z^{\prime}\right)= & N_{T}{ }^{-}\left(z^{\prime}\right)+C^{*}\left[n\left(z^{\prime}\right) / N_{c}\right] \\
& \times\left\{\left[N_{T}\left(z^{\prime}\right)-N_{T}-\left(z^{\prime}\right)\right] / N_{T}\right\} d t .
\end{aligned}
$$

Similarly, transitions involving traps and valence bands lead to time-dependent changes in the holes and occupied trap levels. The results are as follows:

$$
\begin{aligned}
& p_{\text {new }}\left(z^{\prime}\right)=p\left(z^{\prime}\right)-C^{*}\left[p\left(z^{\prime}\right) / N_{v}\right]\left[N_{T}{ }^{-}\left(z^{\prime}\right) / N_{T}\right] d t, \quad(\mathrm{~A} 7 \mathrm{a}) \\
& N_{T}{ }^{-}{ }_{\text {new }}\left(z^{\prime}\right)=N_{T}{ }^{-}\left(z^{\prime}\right)-C^{*}\left[p\left(z^{\prime}\right) / N_{v}\right]\left[N_{T}{ }^{-}\left(z^{\prime}\right) / N_{T}\right] d t,
\end{aligned}
$$

$$
p_{\text {new }}(z)=p(z)+G_{p h}(z)^{*}\left[N_{T}-N_{T}^{-}(z)\right] d t,
$$

and

$$
N_{T}{ }^{-}{ }_{\text {new }}(z)=N_{T}{ }^{-}(z)+G_{p h}(z)^{*}\left[N_{T}-N_{T}{ }^{-}(z)\right] d t .
$$

${ }^{1}$ For example, High-Power Optically Activated Solid-State Switches, edited by A. Rosen and F. J. Zutavern (Artech House, Boston, 1993).

${ }^{2}$ A. Krotkus, S. Marcinkevicius, J. Jasinski, M. Kaminska, H. H. Tan, and C. Jagadish, Appl. Phys. Lett. 66, 3304 (1995).

${ }^{3}$ G. J. Caporaso, Y.-J. Chen, and S. E. Sampayan, Rev. Accel. Sci. Technol. 2, 253 (2009).

${ }^{4}$ K. H. Schoenbach, R. P. Joshi, F. Peterkin, and R. L. Druce, J. Appl. Phys. 77, 5208 (1995)

${ }^{5}$ T. W. Barret, Microwave J. 44, 22 (2001).

${ }^{6}$ R. P. Joshi and D. C. Stoudt, "Photoconductive switches," in Wiley Encyclopedia of Electrical and Electronics Engineering (Wiley, New York, 1999).

${ }^{7}$ P. Kayasit, R. P. Joshi, N. E. Islam, E. Schamiloglu, and J. Gaudet, J. Appl. Phys. 89, 1411 (2001).

${ }^{8}$ Picosecond Optoelectronic Devices, edited by C. H. Lee (Academic Press, New York, 1984).

${ }^{9}$ D. C. Stoudt, K. H. Schoenbach, R. P. Brinkmann, V. K. Lakdawala, and G. A. Gerdin, IEEE Trans. Electron Devices 37, 2478 (1990).

${ }^{10}$ A. S. Weling, B. B. Hu, N. M. Froberg, and D. H. Auston, Appl. Phys. Lett. 64, 137 (1994).

${ }^{11}$ K. H. Schoenbach, J. S. Kenney, F. E. Peterkin, and R. J. Allen, Appl. Phys. Lett. 63, 2100 (1993).

${ }^{12}$ J. C. Adams, R. A. Falk, C. D. Capps, and S. Ferrier, Proc. SPIE 1632, 110 (1992).

${ }^{13}$ F. J. Zutavern, G. M. Loubriel, M. W. O’Malley, L. P. Shanwald, W. D. Helgerson, D. L. McLaughlin, and B. B. McKenzie, IEEE Trans. Electron Devices 37, 2472 (1990).

${ }^{14}$ G. M. Loubriel, F. J. Zutavem, H. P. Hjalmarson, and M. W. O'Malley, in Digest of Technical Papers, 7th IEEE Pulsed Power Conference, Monterey, California, June 1989, edited by R. White and B. H. Bernstein (IEEE, New York, 1989), pp. 365-367.

${ }^{15}$ P. J. Stoudt and M. J. Kushner, J. Appl. Phys. 77, 3518 (1995).

${ }^{16}$ H. Zhao, P. Hadizad, J. H. Hur, and M. A. Gundersen, J. Appl. Phys. 73, 1807 (1993).

${ }^{17}$ L. Hu, J. Su, Z. Ding, Q. Hao, and X. Yuan, J. Appl. Phys. 115, 094503 (2014).

${ }^{18}$ C. R. Crowell and S. M. Sze, Appl. Phys. Lett. 9, 242 (1966).

${ }^{19}$ W. Shockley, Solid State Electron. 2, 35 (1961).

${ }^{20}$ Y. Wang and K. F. Brennan, J. Appl. Phys. 76, 974 (1994).

${ }^{21}$ I. H. Oguzman, Y. Wang, J. Kolnik, and K. F. Brennan, J. Appl. Phys. 77, 225 (1995).

${ }^{22}$ H. W. Thim, J. Appl. Phys. 39, 3897 (1968).

${ }^{23}$ J. B. Gunn, IBM J. Res. Dev. 8, 141 (1964).

${ }^{24}$ B. K. Ridley and T. B. Watkins, Proc. Phys. Soc. 78, 293 (1961).

${ }^{25}$ S. N. Vainshtein, V. S. Yuferev, and J. T. Kostamovaara, J. Appl. Phys. 97, 024502 (2005).

${ }^{26}$ L. Tian and W. Shu, J. Appl. Phys. 103, 124512 (2008).

${ }^{27}$ M. A. Gundersen, J. H. Hur, H. Zhao, and C. W. Myles, J. Appl. Phys. 71, 3036 (1992).

${ }^{28}$ H. Kroemer, Proc. IEEE 53, 1246 (1965).

${ }^{29}$ R. P. Brinkmann, K. H. Schoenbach, D. C. Stoudt, V. K. Lakdawala, G. A. Gerdin, and M. K. Kennedy, IEEE Trans. Electron Devices 38, 701 (1991). 
${ }^{30}$ M. P. Shaw, V. V. Mitin, E. Scholl, and H. L. Grubin, The Physics of Instabilities in Solid State Electron Devices (Plenum Press, New York, 1992).

${ }^{31}$ B. K. Ridley, Proc. Phys. Soc. 82, 954 (1963).

${ }^{32}$ M. A. Lampert and P. Mark, Current Injection in Solids (Academic Press, New York, 1970).

${ }^{33}$ For example, A. G. Milnes, Deep Impurities in Semiconductors (WileyInterscience, New York, 1973).

${ }^{34}$ A. F. Tasch, Jr. and C. T. Sah, Phys. Rev. B 1, 800 (1970).

${ }^{35}$ For example, N. Holonyak, Jr., Proc. IRE 50, 2421 (1962).

${ }^{36}$ K. Kambour, S. Kang, C. W. Myles, and H. P. Hjalmarson, IEEE Trans. Plasma Sci. 28, 1497 (2000).

${ }^{37}$ R. Tiskumara, R. P. Joshi, D. Mauch, J. C. Dickens, and A. A. Neuber, J. Appl. Phys. 118, 095701 (2015).

${ }^{38}$ For example, V. Badescu and P. T. Landsberg, Semicond. Sci. Technol. 8, 1267 (1993).
${ }^{39}$ G. A. Baraff, Phys. Rev. 133, A26 (1964).

${ }^{40}$ L. L. Bonilla, P. J. Hernando, M. Kindelan, and F. Piazza, Appl. Phys. Lett. 74, 988 (1999).

${ }^{41}$ W. Shi, X. Wang, and L. Hou, IEEE Trans. Electron Devices 60, 1361 (2013).

${ }^{42}$ For example, F. J. Zutavern, G. M. Loubriel, M. W. O’Malley, L. P. Schanwald, and D. L. McLaughlin, IEEE Trans. Electron Devices 38, 696 (1991).

${ }^{43}$ R. Courant, K. Friedrichs, and H. Lewy, IBM J. Res. Dev. 11, 215 (1967).

${ }^{44}$ Y. Kiyoyuki, IEEE Electron Device Lett. 8, 73 (1987).

${ }^{45}$ K. Brennan and K. Hess, Phys. Rev. B 29, 5581 (1984).

${ }^{46}$ N. Kroemer, IEEE Trans. Electron Devices 13, 27 (1966).

${ }^{47}$ B. Wang, K. Liu, and J. Qiu, IEEE Trans. Dielectr. Electr. Insul. 20, 1287 (2013).

${ }^{48}$ W. Shi, H. Jiang, M. Li, C. Ma, H. Gui, L. Wang, P. Xue, Z. Fu, and J. Cao, Appl. Phys. Lett. 104, 042108 (2014). 